\title{
Fibrous Dysplasia of the Temporal Bone with Complete Canal Stenosis and Cholesteatoma
}

\author{
Daniel Jethanamest and Pamela Roehm \\ Department of Otolaryngology, New York University School of Medicine, New York, New York \\ U.S.A.
}

Fibrous dysplasia was first described by von Recklinghausen in 1891. Involvement of the temporal bone by this disease process was first reported in $1946(1,2)$. Cases affecting the temporal bone are uncommon, occurring in less than $10 \%$ of all patients (3). Fibrous dysplasia is benign and slowly progressive. Histologically, affected areas demonstrate irregular mixtures of fibrous tissue and mature trabecular bone. Three subtypes of fibrous dysplasia have been described based on the distribution of lesions and associated symptoms. The most common subtype is monostotic fibrous dysplasia which involves a single bone while the polyostotic form involves multiple bones, including the ribs, craniofacial or long bones. McCune-Albright syndrome is the third form and is associated with endocrine abnormalities and café-au-lait spots.

Non-contrast high resolution computed tomography (CT) of the temporal bone is the study of choice for diagnosing fibrous dysplasia in this area given its superior bony detail. Three different appearances of fibrous dysplasia on CT have been described: pagetoid, sclerotic, and cystic (4). The most common of these, the pagetoid pattern, is characterized by a ground-glass appearance on CT imaging. The sclerotic pattern is represented by uniformly dense bone throughout the lesion. Cystic lesions typically include a central radiolucency surrounded by a dense border. Within the temporal bone, the external auditory canal (EAC) is the area most commonly affected by fibrous dysplasia. The EAC is involved in up to $85 \%$ of cases, and manifests as bony protrusions within the EAC or as canal stenosis. (5). We present a unique case of fibrous dysplasia of the temporal bone with early onset complete EAC stenosis presenting as a congenital aural atresia.

A 17-year-old boy presented to our clinic with a lifelong history of right hearing loss and canal atresia. He had no history of otorrhea or otitis media in either ear. On physical exam, he had a dry, atretic right EAC. As revealed by high resolution CT, the stenosis was the result of monostotic fibrous dysplasia limited to the temporal bone (Fig. 1A). The lesion demonstrates the classic pagetoid appearance most commonly found when imaging fibrous dysplasia on CT. Extensive soft tissue density consistent with cholesteatoma was identified in the mesotympanum, surrounding the ossicles (Fig. 1B), despite the presence of a patent Eustachian tube. An associated cholesteatoma is present in nearly $40 \%$ of fibrous dysplasias of the temporal bone. However, the vast majority of these are canal cholesteatomas created

Address for correspondence: Pamela Roehm, M.D., Ph.D., Department of Otolaryngology, New York University School of Medicine, 462 First Avenue, NBV 5E5, New York, New York 10016, pamela.roehm@ nyumc.org. 
by entrapment of the canal skin due to a bony stenosis and limited to the EAC lateral to an intact tympanic membrane $(3,5)$. Our case represents an unusually extensive cholesteatoma extending from the residual canal to involve the middle ear and ossicular chain in the setting of a patent Eustachian tube (Fig. 1C and 1D). The diagnosis in this case was made exclusively by CT. Although observation is often an option in asymptomatic cases, surgical intervention is appropriate in cases with significant functional deficits or complications such as a secondary cholesteatoma formation (1). Our patient had both a functional deficit (complete conductive hearing loss) and a cholesteatoma, which was treated with canalplasty and tympanomastoidectomy. Intraoperatively, we noted the cholesteatoma had eroded the long process of the incus.

The differential diagnosis of bony lesions of the temporal bone includes Paget's disease, which presents with diffuse involvement of the temporal bone and calvarium. Ossifying fibromas, which have a well demarcated border on CT imaging, can also mimic the cystic pattern of fibrous dysplasia. Other bony lesions to consider are exostoses, osteomas, osteochondromas, giant cell granulomas and aneurysmal bone cysts. Patients commonly present with some degree of canal stenosis and an associated progressive conductive hearing loss.

Onset of symptoms in aural atresia is more common within the first two decades of life, and onset in infancy has been reported in different subsites. However, fibrous dysplasia is not typically listed in the differential for congenital aural atresia and though rare should be considered. Patients with functional deficits or suspected cholesteatoma are surgical candidates. Although recurrence after surgery has been reported in up to $49 \%$ of patients, appropriately timed interventions in conjunction with long term follow-up can improve hearing loss and prevent complications $(1,5)$.

\section{REFERENCES}

1. Kim YH, Song J, Choi HG, et al. Role of surgical management in temporal bone fibrous dysplasia. Acta Otolaryngol. 2009; 129(12):1374-1379. [PubMed: 19922084]

2. Lustig LR, Holliday MJ, McCarthy EF, Nager GT. Fibrous dysplasia involving the skull base and temporal bone. Arch. Otolaryngol. Head Neck Surg. 2001; 127(10):1239-1247. [PubMed: 11587606]

3. Zanetti D, Gamba P. Cholesteatoma and fibrous dysplasia of the temporal bone: case report and review of the literature. J Otolaryngol. 2007; 36(1):59-63. [PubMed: 17376353]

4. Brown EW, Megerian CA, McKenna MJ, Weber A. Fibrous dysplasia of the temporal bone: imaging findings. AJR Am J Roentgenol. 1995; 164(3):679-682. [PubMed: 7863893]

5. Megerian CA, Sofferman RA, McKenna MJ, Eavey RD, Nadol JB. Fibrous dysplasia of the temporal bone: ten new cases demonstrating the spectrum of otologic sequelae. Am J Otol. 1995; 16(4):408-419. [PubMed: 8588639] 


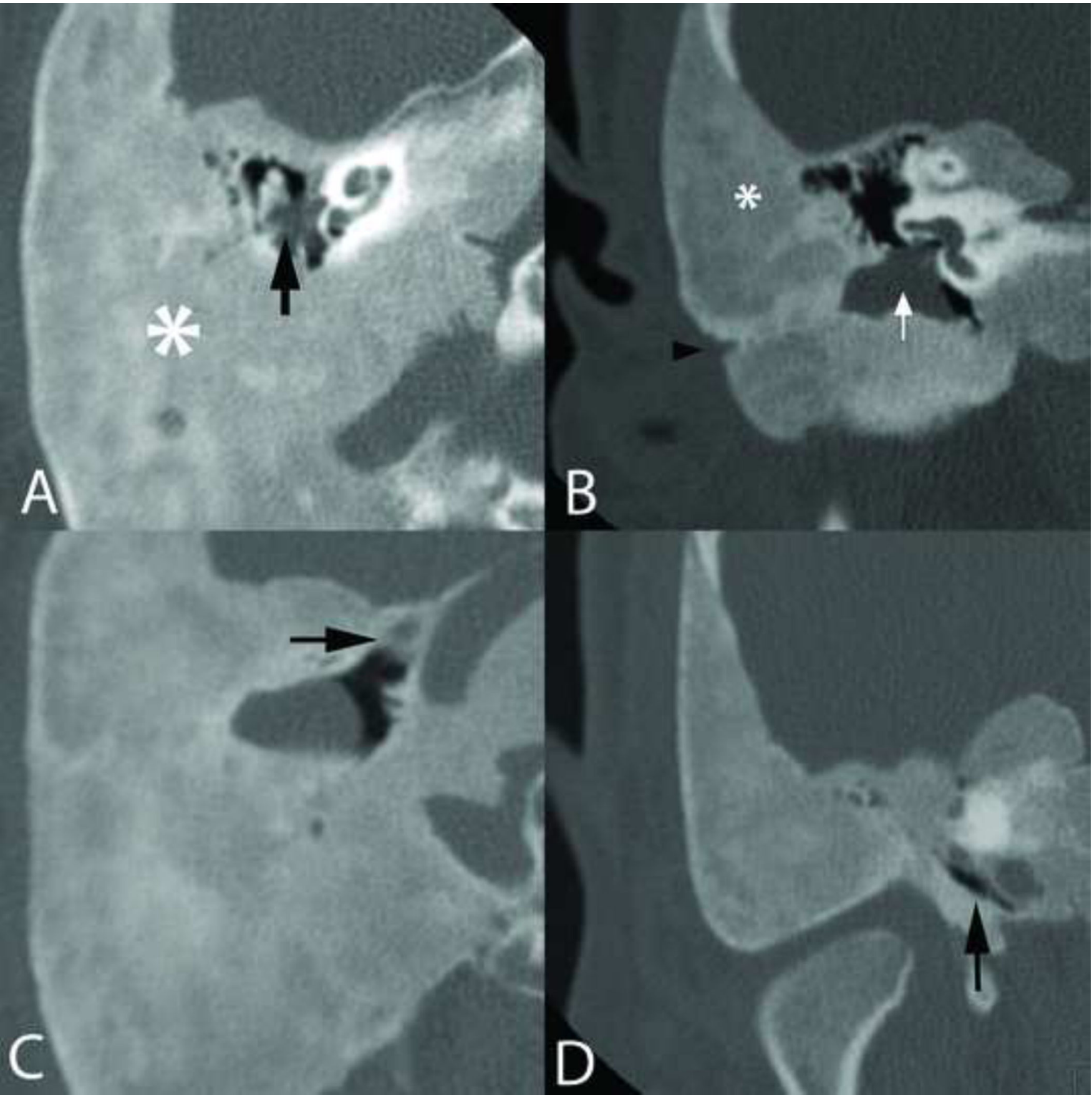

Figure 1.

(A) Axial high resolution temporal bone CT through the plane of the cochlea. CT imaging reveals a pagetoid pattern of bony development (asterisk), with a focus of soft tissue density within the middle ear space adjacent to the ossicular chain (arrow). (B) Coronal CT reconstruction in the plane of the vestibule and external auditory canal. Complete stenosis of the ear canal is noted with only a vestigial external opening of the canal present (arrowhead) encased by the pagetoid dysplastic bone (asterisk). Cholesteatoma within the medial remnant of the external auditory canal and middle ear is clearly visible (arrow). (C) Axial 
and (D) coronal CT at the level of the proximal Eustachian tube (black arrows), displaying patency without stenosis or obstruction. 